\title{
Low-temperature specific heat of nanotube systems
}

\author{
Valentin N. Popov* \\ Physics Department, University of Antwerp (RUCA), 2020 Antwerp, Belgium \\ (Received 20 March 2002; revised manuscript received 28 May 2002; published 29 October 2002)
}

\begin{abstract}
The low-temperature specific heat of single-walled carbon nanotubes (isolated and bundled) and multiwalled carbon nanotubes is calculated within force-constant dynamical models. It is shown that, due to the quadratic dependence of the frequency $\omega$ of the transverse-acoustic phonons on the wave number $q$, the phonon density of states has a singularity of the type $1 / \sqrt{\omega}$ and the very low-temperature specific heat varies as $\sqrt{T}$ with the temperature $T$. With the increase of the diameter of the system, the contributions of the longitudinal- and torsional-acoustic phonons to the specific heat begin to prevail and the latter becomes linear in $T$. These results are confirmed by the recent experimental data.
\end{abstract}

DOI: 10.1103/PhysRevB.66.153408

PACS number(s): 63.22. $+\mathrm{m}, 65.90 .+\mathrm{i}$

The first observation of multiwalled nanotubes (MWNT's) was reported in $1991 .{ }^{1}$ Soon after that, singlewalled nanotubes (SWNT's) were produced in large amounts. ${ }^{2}$ Later, bundles of hundreds of tubes with nearly uniform diameters of $\approx 1.38 \mathrm{~nm}$ were synthesized in high yields. ${ }^{3}$ Finite nanotube bundles have unique physical properties due to their quasi-one-dimensionality. ${ }^{4}$ In particular, these systems have four vibrational acoustic branches while only three such branches exist in three-dimensional (3D) systems. As a consequence, the phonon contribution to the lowtemperature specific heat of nanotube systems is expected to change with the temperature $T$ as $T^{n}$ with $n \leqslant 3 .^{5}$

The low-temperature specific heat has been the goal of several experimental studies in the last few years. The specific heat of MWNT's measured by Yi et al. ${ }^{6}$ from 10 to 300 $\mathrm{K}$ was found to have linear temperature dependence over the entire temperature interval. This was attributed to a constant acoustic-phonon density of states of the MWNT's which could originate from the acoustic branches of the layers alone. It was concluded that the coupling between the layers was much weaker than in graphite. The turbostratic stacking of the adjacent layers and the larger interlayer separations than in graphite were found responsible for the weak interlayer coupling. In Ref. 7, the results of the measurements of the specific heat of MWNT's and bundles of SWNT's with average tube diameter of $1.3 \mathrm{~nm}$ in the temperature range 1 $<T<200 \mathrm{~K}$ were presented. The MWNT sample data had graphite-like behavior, while the bundle sample data had surprisingly strong temperature dependence at low temperatures. In order to explain the bundle data, a bundle was modeled by a hexagonal array of interacting linear chains of rings, each chain having four linear acoustic branches. The results of this simple model significantly underestimated the experimental data. It was concluded that the steep $T$ dependence of the specific heat of bundles could be due to the presence of impurities in the sample. In Ref. 8, the specific heat of bundles of SWNT's with the average tube diameter of $1.25 \mathrm{~nm}$, measured from $300 \mathrm{~K}$ down to $2 \mathrm{~K}$, was reported. The experimental data were found to lie close to the theoretical curve for SWNT's above $4 \mathrm{~K}$, and to differ remarkably from the curves for graphene and graphite up to about $100 \mathrm{~K}$. Based on an anisotropic two-band Debye model with linear acoustic dispersion, it was argued that the intertube interactions in a bundle should be very weak possibly due to the different types of the neighboring tubes. Recently, the specific heat of SWNT bundles was measured down to $0.1 \mathrm{~K} .{ }^{9}$ The corrected data were fitted with the combination of power laws $0.043 T^{0.62}+0.035 T^{3}$, where the first term could not be explained neither with electronic, nor with disorder contributions to the specific heat. The models considered in Refs. 7 and 8, in view of the approximations made, cannot provide an exact description of the temperature dependence of the specific heat and, consequently, there is an obvious necessity for its systematic theoretical study.

Here, the low-temperature (LT) specific heat of SWNT's (isolated and bundled) and of MWNT's is studied within force-constant dynamical models that take into account the atomic structure of the nanotubes. These models have already been used to calculate the low-frequency dynamics of bundles of SWNT's (Ref. 10) and MWNT's. ${ }^{11}$ A bundle of SWNT's is modeled by several identical tubes arranged in a close-packed structure, and a MWNT is modeled by several coaxial layers. In both cases, a tube or a layer can be viewed as obtained by rolling of an infinite strip of a graphite sheet into a seamless cylinder and can uniquely be specified by a pair of integers $(n, m), n \geqslant m \geqslant 0$. The dynamical models use the one-dimensional periodicity of the tubes/layers and can handle only bundles/MWNT's with tubes/layers of commensurate unit-cell lengths. The intratube/intralayer carboncarbon interactions are described by force constants of the valence force field (VFF) type. ${ }^{10,11}$ The use of fixed force constants instead of pair potentials rules out the possibility for structural optimization of the tubes/layers. For this reason, all carbon-carbon bond lengths are assumed equal to $1.42 \AA$ as in graphite and close to the average value measured in nanotubes, and all bond angles are assumed equal to each other. The intertube/interlayer carbon-carbon interactions are modeled by a potential of the Lennard-Jones (LJ) type. The interaction energy for a given bundle of SWNT's or a MWNT is obtained by summing over all pairs of atoms belonging to different tubes/layers. This energy is then minimized with respect to the intertube/interlayer separations, the relative angles of rotation of the tubes/layers around their axes and the relative translations of the tubes/layers along the same axes. In the case of MWNT's, it is implied that the adjacent layers must be of type for which the initial radii 
difference is close to the optimized interlayer separation, so that the change of the bond lengths is small. For the optimized structure, the dynamical matrix is calculated by the use of the VFF parameters and the LJ potential. The vibrational modes of the system are then derived as solutions of the dynamical eigenvalue problem. More details can be found in Refs. 10 and 11. Finally, the specific heat is obtained by approximating the integral over the Brillouin zone (see below) with a sum over 200 points in one-half of the 1D Brillouin zone for SWNT's, MWNT's, and bundles of SWNT's and over 1600 points in the irreducible wedge of the hexagonal Brilloiun zone for infinite bundles of SWNT's. The calculations of the specific heat were carried out with the entire phonon dispersion for temperatures from 0 to $4000 \mathrm{~K}$. Since the measured specific-heat curves for various nanotube systems differ significantly only below $100 \mathrm{~K},{ }^{8}$ the calculated curves are given below only for $0<T<100 \mathrm{~K}$. We note, that in view of the restrictions on the type of the tubes/ layers which can be handled within the models used here, the stacking of the tubes/layers will be close to that in graphite and the intertube/interlayer interactions will be strong. Therefore, the results of these models will correspond to the so-called strong-coupling regime.

The main contribution to the specific heat of nanotube systems is the vibrational one because the electronic one is negligible even at a few Kelvin. ${ }^{5}$ In this study, the electronic specific heat will be ignored and, therefore, the dependence of the specific heat of nanotube systems at constant volume $C_{v}$ on the temperature $T$ is given by

$$
C_{v}(T)=k_{B} \int \frac{\left(\hbar \omega / k_{B} T\right)^{2} \exp \left(\hbar \omega / k_{B} T\right)}{\left[\exp \left(\hbar \omega / k_{B} T\right)-1\right]^{2}} D(\omega) d \omega,
$$

where $D(\omega)$ is the phonon density of states (PDOS). The high-temperature (or classical) limit of this expression does not depend on the particular structure of the carbon system and is equal to $3 k_{B} / m \approx 2078 \mathrm{~mJ} / \mathrm{gK}$ with $m$ being the atomic mass of carbon. The LT behavior of $C_{v}$ is closely connected to the dimensionality of the system. For low enough temperatures, when the population of the lowest optical branches can be ignored, the specific heat is determined by the acoustic branches alone. If $\omega_{o}$ is the frequency of the lowest-energy optical phonon, then the optical phonons will give negligible contribution to the specific heat for temperatures less than $T_{o} \approx \hbar \omega_{o} / 6 k_{B}$ for which the factor multiplying $D(\omega)$ becomes smaller than $0.1 .^{7}$ In the interval $0<T$ $<T_{o}, C_{v}(T)$ can readily be obtained from the expression above once the acoustic-phonon dispersion is known. For the 3D system of graphite, for any of the three acoustic branches $\omega \propto q$, therefore, $D(\omega) \propto \omega^{2}$ and $C_{v} \propto T^{3}$. For the 2D system of graphene, for the in-plane longitudinal-acoustic (LA) and transverse-acoustic (TA) phonons $\omega \propto q$, so that $D(\omega) \propto \omega$ and $C_{v} \propto T^{2}$; for the out-of-plane acoustic (ZA) phonons $\omega$ $\propto q^{2}, D(\omega)=$ const and $C_{v} \propto T$ (see Table I).

In the case of isolated SWNT's, two force-constant models ${ }^{12,13}$ yielded four acoustic branches with linear $q$ dependence of $\omega$. Similar dispersion was obtained within a tight-binding $^{14}$ and an $a b$ initio $^{15}$ approaches. It is now
TABLE I. Low-temperature specific-heat behavior of the 3D, 2D, and 1D systems of graphite, graphene, and SWNT, respectively. For finite bundles of SWNT's and MWNT's, the specific heat will have the same behavior as for isolated SWNT's.

\begin{tabular}{lcccc}
\hline \hline System & $\begin{array}{c}\text { Acoustic } \\
\text { branch }\end{array}$ & $\begin{array}{c}\text { Phonon } \\
\text { dispersion }\end{array}$ & Phonon DOS & Specific heat \\
\hline Graphite & LA, TA & $\omega \propto q$ & $D(\omega) \propto \omega^{2}$ & $C_{v} \propto T^{3}$ \\
Graphene & LA, TA & $\omega \propto q$ & $D(\omega) \propto \omega$ & $C_{v} \propto T^{2}$ \\
& ZA & $\omega \propto q^{2}$ & $D(\omega)=$ const & $C_{v} \propto T$ \\
SWNT & LA, TW & $\omega \propto q$ & $D(\omega)=$ const & $C_{v} \propto T$ \\
& TA & $\omega \propto q^{2}$ & $D(\omega) \propto 1 / \sqrt{\omega}$ & $C_{v} \propto \sqrt{T}$ \\
\hline \hline
\end{tabular}

widely accepted that the frequency of all four acoustic branches has linear $q$ dependence. ${ }^{16}$ In a systematic study of the elastic properties of isolated SWNT's (Ref. 17), it was argued that the frequency of the transverse-acoustic modes must be quadratic in $q$. This frequency dependence has crucial consequencies for the LT specific heat. Indeed, while for the LA and torsional-acoustic (TW) phonons $\omega \propto q, D(\omega)$ $=$ const and $C_{v} \propto T$, for the TA phonons $\omega \propto k^{2}, D(\omega)$ $\propto \omega^{-1 / 2}$ and $C_{v} \propto T^{1 / 2}$ (see Table I). The temperature interval of applicability of these power laws for the specific heat is again determined by the lowest-frequency optical phonon. For example, for a SWNT $(10,10), \omega_{o} \approx 20 \mathrm{~cm}^{-1} \approx 29 \mathrm{~K}$ and, therefore, $T_{o} \approx 5 \mathrm{~K}$. The calculated PDOS of isolated SWNT's (see Fig. 1) has a characteristic singularity of the type $1 / \sqrt{\omega}$ at $\omega=0$, while graphene has finite PDOS and graphite has zero PDOS at this frequency.

The finite bundles of SWNT's are 1D systems and for them apply the theoretical results for isolated SWNT's (see Table I). In order to obtain quantitative results for bundles, the behavior of $C_{v}(T)$ is studied for bundles with different

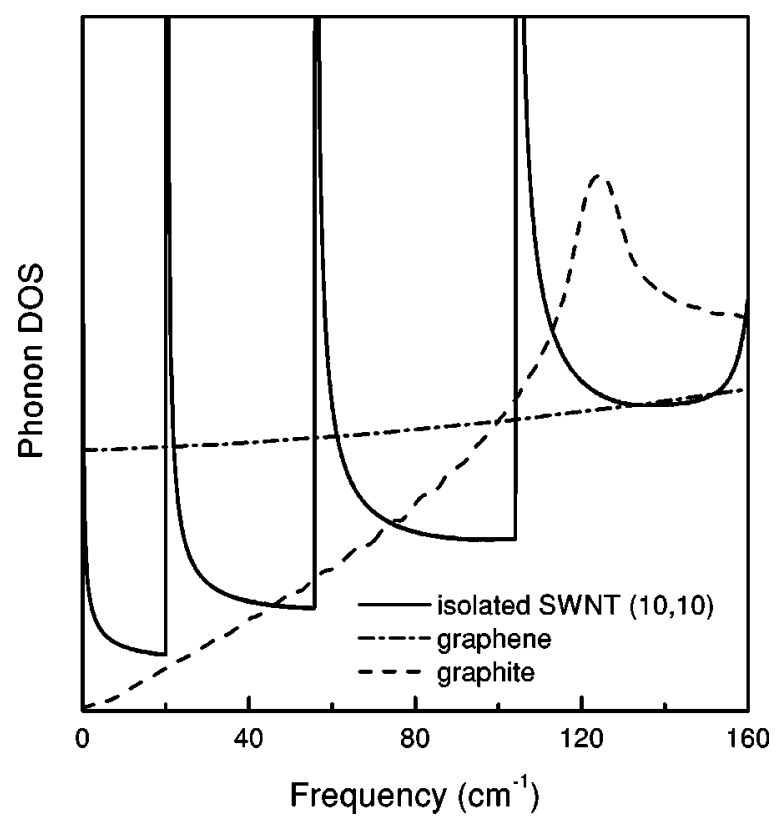

FIG. 1. Calculated phonon density of states of an isolated SWNT $(10,10)$, graphene, and graphite. Notice the spike at $\omega=0$ due to the TA phonons of the nanotube. 


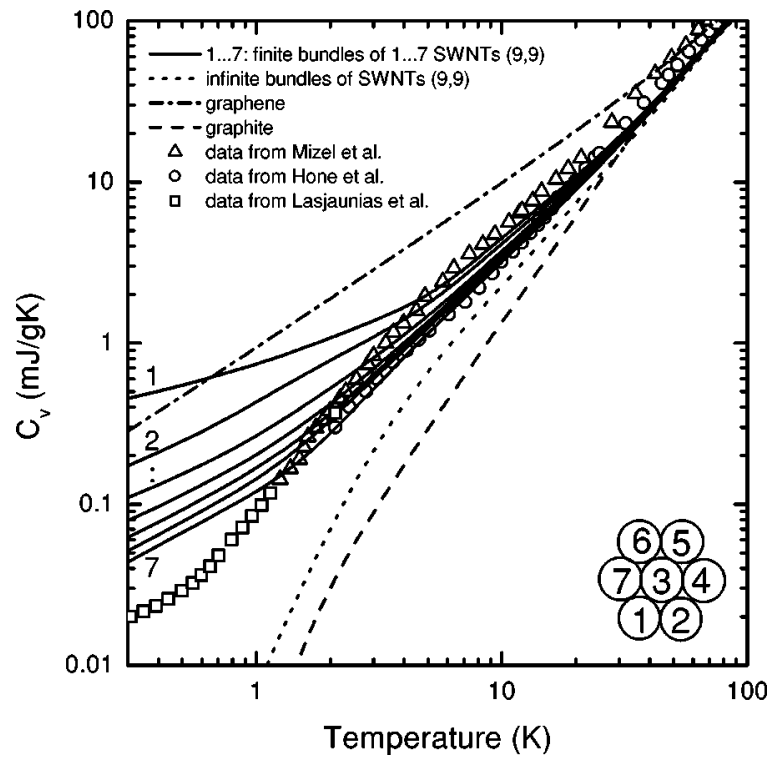

FIG. 2. Calculated specific heat for finite bundles of 1 to 7 SWNT's $(9,9)$, infinite bundles of such nanotubes, and graphite and graphite are shown in comparison with the available experimental data on bundles of nanotubes. The figure in the right corner depicts the construction of the bundle. On adding tubes to the bundle, the temperature dependence of the specific-heat changes to linear. Note that for graphene, $C_{v} \propto T$ in the entire displayed interval, while for graphite $C_{v} \propto T^{3}$ below $\approx 11 \mathrm{~K}$. For infinite bundles, $C_{v} \propto T^{3}$ only below $\approx 3 \mathrm{~K}$.

number $n$ of SWNT's $(9,9)(n=1,2, \ldots, 7)$ within the model described above. The calculated $C_{v}(T)$ is compared to the available experimental data in Fig. 2. First, it is seen that the specific heat of an isolated SWNT $(9,9)$ exhibits three different regimes for $T<100 \mathrm{~K}$. For very low temperatures, only TA phonons are excited so that $C_{v} \propto T^{1 / 2}$ (slope $1 / 2$ on the $\log -\log$ plot in Fig. 2). With the increase of $T$, the contribution of LA and TW phonons to $C_{v}$ becomes larger than that of the TA phonons in favor of $C_{v} \propto T$ (slope 1). Finally, above $T \approx 5 \mathrm{~K}$, the optical phonons begin to contribute to the specific heat and its $T$ dependence is modified again. We note that similar temperature dependence may be expected for the thermal conductivity that is predominantly phononic and in a certain approximation is proportional to the specific heat. Second, the $\sqrt{T}$ part of $C_{v}(T)$ (slope 1/2) diminishes when tubes are added to the bundle. The reason for this is that the slope of the TA branches (i.e., the group velocity) is proportional to the bundle radius [Eq. (2.22) in Ref. 17)]. Therefore, with the increase of the bundle size, the relative contribution to $C_{v}$ of the TA branches at a given temperature decreases. For $N=7$, the $\sqrt{T}$ part is not observable and the LT specific heat is linear with $T$ because of the dominant contributions of LA and TW phonons. With the increase of the bundle size, the specific heat is expected to tend to that of infinite bundles. The LT part of the specific heat of infinite bundles and graphite vary as $T^{3}$, but the former excedes the latter because of the weaker interactions between the tubes in comparison with the interaction between the graphite sheets. It is seen in Fig. 2 that with the increase of the number of tubes in the bundle from 1 to 7 , the calculated $C_{v}(T)$ for

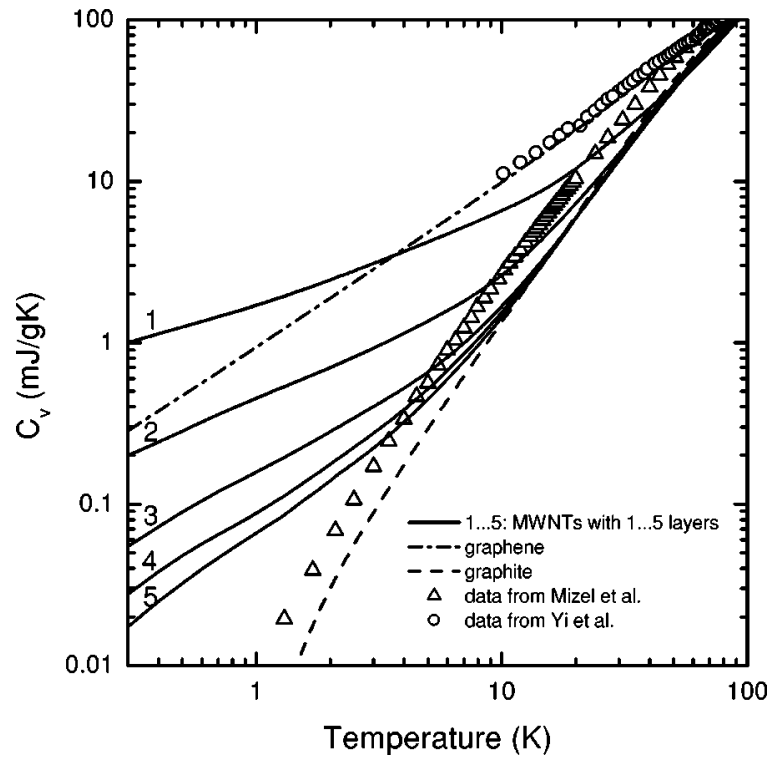

FIG. 3. Calculated specific heat for MWNT's $(5,5)$, $(5,5) @(10,10), \ldots,(5,5) @ \ldots .(25,25)$ in comparison with available experimental data on MWNT's. With the increase of the number of layers, the temperature dependence of the specific-heat changes to linear. The specific heat of a MWNT in the limit of large number of layers tends to that of graphite.

finite bundles of SWNT's tends to the experimental points of Ref. 8 as an indication for good stacking of the tubes in the sample. The calculational results, however, underestimate the data of Ref. 7. Our predictions agree qualitatively well with recent specific-heat data on bundles measured down to $0.1 \mathrm{~K}$ and fitted with $0.043 T^{0.62}+0.035 T^{3} .{ }^{9}$ The power of the first term, being less than unity, cannot be explained with the assumption of linear acoustic phonon dispersion only. In view of the theoretical results above, we argue that the first term is due to contributions from all acoustic branches (LA and TW with linear dispersion and TA with quadratic dispersion).

The specific heat of MWNT's, being 1D systems, must behave like that of isolated SWNT's (see Table I). It may be expected that with the increase of the number of layers, the TA branches will harden and the dominant contribution of LA and TW phonons will result in linear specific heat. To check this suggestion, the specific heat of a five-walled nanotube $(5,5) @(10,10) @, \ldots,(25,25)$ was calculated. It can be seen in Fig. 3 that starting from a single layer $(5,5)$ and adding more layers to it, the $\sqrt{T}$ part diminishes and disappears and is replaced by a linear $T$ dependence. For many layers, the specific heat is expected to tend to that of graphite. The calculated curves for MWNT's differ significantly from the experimental data ${ }^{6,7}$ possibly due to the reduced vibronic coupling in the measured MWNT's.

The case of very large-size bundles of SWNT's and MWNT's cannot be treated within the atomistic approach due to computational limitations. However, the behavior of $C_{v}(T)$ can be deduced from general arguments. Indeed, for small diameter-length ratio, a bundle can be considered as a thin rod which has linear dispersion of the longitudinal and torsional elastic waves, whereas the dispersion of the trans- 
verse elastic waves is quadratic. Since practically the measured bundles can be modeled by thin rods, the observed LT specific heat will be linear with $T$. The temperature interval for this behavior will decrease with the increase of the number of tubes in the bundle and the number of layers in the MWNT due to the contribution of very low-energy optical phonons to $C_{v}$.

In summary, calculations of the specific heat of SWNT's (isolated and bundled) and MWNT's were performed within an approach that accounts for the atomic structure of these systems. For finite bundles of SWNT's and for MWNT's, the quadratic $q$ dependence of the frequency of the TA phonons leads to a $1 / \sqrt{\omega}$ singularity of the phonon density of states, which gives rise to a $\sqrt{T}$ dependence of the very LT specific heat. The adding of tubes to a bundle or layers to a MWNT

*Permanent address: Faculty of Physics, University of Sofia, BG1164 Sofia, Bulgaria.

${ }^{1}$ S. Iijima, Nature (London) 354, 56 (1991).

${ }^{2}$ S. Iijima and T. Ichihashi, Nature (London) 363, 603 (1993); D. S. Bethune, C. H. Kiang, M. S. de Vries, G. Gorman, R. Savoy, J. Vazquez, and R. Beyers, ibid. 363, 605 (1993).

${ }^{3}$ A. Thess, R. Lee, P. Nikolaev, H. Dai, P. Petit, J. Robert, C. Xu, Y. H. Lee, S. G. Kim, A. G. Rinzler, D. T. Colbert, G. E. Scuseria, D. Tománek, J. E. Fischer, and R. E. Smalley, Science 273, 483 (1996).

${ }^{4}$ M. S. Dresselhaus, G. Dresselhaus, and Ph. Avouris, Carbon Nanotubes: Synthesis, Structure, Properties and Applications, Topics in Applied Physics Vol. 80 (Springer-Verlag, Berlin, 2001).

${ }^{5}$ L. X. Benedict, S. G. Louie, and M. L. Cohen, Solid State Commun. 100, 177 (1996).

${ }^{6}$ W. Yi, L. Lu, Zhang Dian-lin, Z. W. Pan, and S. S. Xie, Phys. Rev. B 59, R9015 (1999).

${ }^{7}$ A. Mizel, L. X. Benedict, M. L. Cohen, S. G. Louie, A. Zettl, N. K. Budraa, and W. P. Beyermann, Phys. Rev. B 60, 3264 (1999). suppresses this behavior in favor of linear temperature dependence. Finally, the enlargement of the bundles/MWNT's will result in a crossover to a 3D behavior. The predictions for $C_{v}(T)$ of bundles are in fair agreement with two sets of experimental data ${ }^{8,9}$ in favor of the relatively good stacking of the tubes in the samples. On the other hand, our results disagree with the bundle data of Ref. 7 and the MWNT data of Refs. 6 and 7, which can be considered as an indication for the relative weakness of intertube/interlayer interactions in the samples.

The author acknowledges financial support by the Flemish Region-IWT and by NATO. He also thanks the University of Antwerp (RUCA) for its support in the framework of the Visiting Professors Program.

${ }^{8}$ J. Hone, B. Batlogg, Z. Benes, A. T. Johnson, and J. E. Fischer, Science 289, 1730 (2000).

${ }^{9}$ J. C. Lasjaunias, K. Biljaković, Z. Benes, J. E. Fischer, and P. Monceau, Phys. Rev. B 65, 113409 (2002).

${ }^{10}$ V. N. Popov, V. E. Van Doren, and M. Balkanski, Solid State Commun. 114, 395 (2000); V. N. Popov and L. Henrard, Phys. Rev. B 63, 233407 (2001).

${ }^{11}$ V. N. Popov and L. Henrard, Phys. Rev. B 65, 235415 (2002).

${ }^{12}$ R. Saito, T. Takeya, T. Kimura, G. Dresselhaus, and M. S. Dresselhaus, Phys. Rev. B 57, 4145 (1998).

${ }^{13}$ J. Maultzsch, S. Reich, C. Thomsen, E. Dobardžić, I. Milošević, and M. Damnjanović, Solid State Commun. 121, 471 (2002).

${ }^{14}$ J. Yu, R. K. Kalia, and P. Vashishta, J. Chem. Phys. 103, 6697 (1995).

${ }^{15}$ D. Sánchez-Portal, E. Artacho, J. M. Soler, A. Rubio, and P. Ordejón, Phys. Rev. B 59, 12678 (1999).

${ }^{16}$ M. S. Dresselhaus and P. C. Eklund, Adv. Phys. 49, 705 (2000).

${ }^{17}$ V. N. Popov, V. E. Van Doren, and M. Balkanski, Phys. Rev. B 61, 3078 (2000). 